\title{
Problematische Verstrickungen eines Kindertherapeuten
}

\section{Zu: Helmut Wetzel: Das Hilfeersuchen des Kindes in der Therapie: Subjektive Wirklichkeiten und kommunikative Strukturen. Verhaltenstherapie 2010;20:53-59}

\author{
Lutz Goldbeck $^{\mathrm{a}}$ Franz Petermann ${ }^{\mathrm{b}}$ \\ ${ }^{a}$ Klinik für Kinder- und Jugendpsychiatrie/Psychotherapie, Universitätsklinikum Ulm, \\ ${ }^{\mathrm{b}}$ Zentrum für Klinische Psychologie und Rehabilitation, Universität Bremen, Deutschland
}

Interventionen bei Kindesmisshandlung und sexuellem Missbrauch von Kindern stellen alle beteiligten Professionellen angesichts der Komplexität psychologischer, medizinischer, rechtlicher, sozialer und ethischer Aspekte des Kinderschutzes vor große Herausforderungen. Die Ausbildung von Kinder- und Jugendlichenpsychotherapeuten und Psychologischen Psychotherapeuten in Kinderschutzbelangen ist genauso wie diejenige von Ärzten oder Lehrern und Erziehern oft lückenhaft; systematische Anleitungen sind dringend erforderlich [vgl. Bretz et al., 1997]. Insgesamt stehen zu wenig evidenzbasierte Methoden zur Verfügung, das Problem erscheint zu schwierig und provoziert entweder Vermeidung oder blindes Agieren.

Wetzel stellt in Heft 1/2010 dieser Zeitschrift in seinem Beitrag in der Rubrik «Für die Praxis» wesentliche Fragen nach der Rolle und dem Auftrag von Kindertherapeuten, wenn sich bei der Konsultation oder im Therapieverlauf Hinweise auf eine intrafamiliäre Kindeswohlgefährdung ergeben. Er möchte mit seinen Reflexionen über eigene Erfahrungen «zu einem besseren Verständnis von misshandelten oder missbrauchten Kindern beitragen und für die praktische therapeutische Arbeit nützlich sein.» (p 53). Leider erreicht der Autor mit seinem Beitrag, einer Mischung aus persönlichen Meinungen, Fragmenten aus Verbatim-Transkripten von Interaktionen mit Patientenfamilien, Beschreibungen und Deutungen von Kinderzeichnungen sowie vagen Hinweisen auf ein Praxisforschungsprojekt ungewollt das Gegenteil: Er demonstriert am eigenen Beispiel die problematische Verstrickung und Rollenkonfusion, in die Therapeuten geraten können, wenn ihre Rolle und Aufgabe nicht klar definiert sind und sie keine evidenzbasierten Methoden zur Verfügung haben, um Kindeswohlgefährdungen der ihnen anvertrauten Kinder aufzudecken und Kinderschutz bei Bedarf interdisziplinär zu organisieren. So trägt die in hohem Maße «subjektive Wirklichkeit» des Autors mehr zur Verwirrung von Praktikern und zur Vernebelung des Phänomens als zur Hilfestellung für praktisch tätige Verhaltenstherapeuten bei.

Befremdlich sind zunächst die unklaren und allenfalls impliziten Fragestellungen und die Zielsetzung des Beitrags. Es fällt schon beginnend mit dem Titel eine durchgängig schwammige Terminologie (z.B. «Struktur» in allen möglichen Zusammensetzungen), der Verzicht auf Definitionen von zentralen Begriffen und das Fehlen eines Theoriebezugs oder einer wissenschaftlichen Basierung der getroffenen Aussagen auf. Die genannten Thesen bleiben somit ohne Beleg und sind überwiegend unverständlich. Die Fallvignetten lassen eine verhaltensnahe Deskription vermissen und die «Befunde» («ausgesprochen sympathischer und netter Junge», p 54) sowie die dazu mitgeteilten Deutungen und Kommentare sind in hohem Maße wertend und schwer nachvollziehbar. Metaphern, die wenn überhaupt in der Supervision zur Stimulation von freier Assoziation nützlich sein mögen («Aus der Perspektive des Balls ist Tennis ein mörderisches Spiel», p 54), wirken im vorliegenden Beitrag nicht zielführend. Wie der Therapeut/ Autor im konkreten Fall von «Tom» überhaupt Verdacht schöpft, der Junge könne von seinem Stiefvater misshandelt worden sein, erschließt sich dem Leser nicht. Dabei wird ja an anderer Stelle richtigerweise postuliert, dass allein die Aussage des Kindes hierzu Informationen liefern kann. Stattdessen wird im konkreten Fallbeispiel die Misshandlung als Fakt

\section{KARGER}

Fax +497614520714

Information@Karger.de

www.karger.com (c) 2010 S. Karger GmbH, Freiburg

Accessible online at:

www.karger.com/ver
Prof. Dr. Lutz Goldbeck

Universitätsklinik für Kinder- und Jugendpsychiatrie und Psychotherapie Steinhövelstraße 5

89075 Ulm, Deutschland

Lutz.Goldbeck@uniklinik-ulm.de
Prof. Dr. Franz Petermann

Zentrum für Klinische Psychologie und Rehabilitation der Universität Bremen

Grazer Straße 2 und 6 28359 Bremen, Deutschland fpeterm@uni-bremen.de 
unterstellt («gewaltsam zum Schweigen gebrachtes Kind», p 54) und vage Spekulationen werden verbalisiert. Besonders verwirrend sind Widersprüche in zentralen Aussagen und Empfehlungen des Autors innerhalb des Beitrags selbst, z.B. «Zielrichtung der Therapie ist nicht der objektive Tatbestand oder die Wahrheitsfindung» (p 59) versus «Das Ziel der Wahrheitsfindung steht nicht allein im Vordergrund» (ebda.). Eine Trennung von forensischer Evaluation, psychologischer Glaubhaftigkeitsbeurteilung von Zeugenaussagen und Psychotherapie etwaiger Traumafolgestörungen (optimalerweise in dieser Reihenfolge) ist jedoch unerlässlich, und die Verwertbarkeit von im therapeutischen Kontext dokumentiertem Verhalten von Kindern als «Opferzeugen» vor Gericht ist aus diversen Gründen (Schweigepflicht, Suggestionseffekte; vgl. Heubrock und Petermann [1998]) problematisch.

Bedauerlicherweise scheitert der Autor mit Hinblick auf die Brisanz des Themas Kindesmisshandlung und der hohen Dunkelziffer gefährdeter Kinder an seinem selbst gesetzten Anspruch, das Thema einzuordnen und hilfreiche Handlungsempfehlungen für Therapeuten auszusprechen. Der Autor bleibt auf der Ebene der Mitteilung eigener Erfahrungen und Schwierigkeiten stehen.

Wie sich in einer Interventionsstudie zur Überprüfung eines externen Hilfeprozess-Management-Ansatzes mit Sozialarbeitern und Therapeuten gezeigt hat [vgl. Goldbeck et al., 2007], ist gerade die subjektive Gewissheit von Praktikern («Jetzt erschließt sich auch mir der Sinn all dieser Striche und Figuren», p 55) im Umgang mit möglicherweise misshandelten Kindern ein Hindernis bei der Klärung von Verdachtsfällen und bei der Organisation wirksamer Hilfen. Einschlägige Fachgesellschaften haben im Konsensusverfahren und auf der Basis beginnender empirischer Forschung im Kinderschutz (letztere erfolgt leider bislang vor allem im angloamerikanischen Raum) Leitlinien für Therapeuten [vgl. Fegert et al., 2007; Practice Parameter der AACAP, 1997] und Kinderärzte [Kellogg et al., 2005] verfasst, auf die wenigstens hingewiesen werden sollte. Klopfer et al. [1999] haben in ihrem Forschungsprojekt institutionelle Reaktionen auf Missbrauch in Institutionen, die Tragik der Rollenkonfusion von Therapeu- ten, die ermitteln wollen, und Polizisten, die vorwiegend helfen wollen, bereits beschrieben und ein besseres Zusammenwirken der Professionellen mit den für Kinderschutz und für die Hilfeplanung zuständigen Jugendämtern angemahnt.

Hoffentlich wird sich durch die inzwischen breiter gewordene gesellschaftliche Debatte um Kindesmisshandlung und sexuellen Missbrauch von Kindern und um die fatalen Folgen von Traumatisierung im Kindesalter auch in der Berufsgruppe der Psychotherapeuten künftig ein stärkeres Bedürfnis nach Fortbildung und Evidenzbasierung des eigenen Handelns in diesem Bereich manifestieren. Es bleibt das Verdienst der Zeitschrift Verhaltenstherapie, mit dem Sonderheft ein Thema angestoßen zu haben, das wegen seines «Schmuddel»-Images und seiner vermeintlichen Unwissenschaftlichkeit bislang von der Psychotherapieforschung und von Klinikern vernachlässigt wurde.

\section{Literatur}

AACAP (ed): Practice parameters for the forensic evaluation of children and adolescents who may have been physically or sexually abused. J Am Acad Child Adolesc Psychiatry 1997;36:423-442.

Bretz E, Richter N, Petermann F, Waldmann HC: Entwicklung und Evaluation eines Fragebogens zur Evaluation einer Erzieherinnenfortbildung zum Thema sexueller Missbrauch. Praxis Kinderpsychol Kinderpsychiatr 1997;46:420-434.

Fegert JM, Frank R, Goldbeck L, Höhne D, Schepker R: Vernachlässigung, Misshandlung, sexueller Missbrauch; in Deutsche Gesellschaft für Kinder- und Jugendpsychiatrie, Psychotherapie und Psychosomatik, Bundesarbeitsgemeinschaft Leitender Klinikärzte für Kinder- und Jugendpsychiatrie, Psychotherapie und Psychosomatik, Berufsverband der Ärzte für Kinder- und Jugendpsychiatrie, Psychotherapie und Psychosomatik (Hrsg): Leitlinien zu Diagnostik und Therapie von psychischen Störungen im Säuglings-, Kindes- und Jugendalter, ed 3. Köln, Deutscher Ärzte-Verlag, 2007, pp 423-435.

Goldbeck L, Laib-Koehnemund A, Fegert JM: A randomized controlled trial of consensus-based child abuse case management. Child Abuse Negl 2007;31:919-33. Heubrock D, Petermann F: Neuropsychological assessment of suspected malinge-

ring: Research results, evaluation techniques and further directions of research and application. Eur J Psychol Assess 1998;14:211-225.

Kellogg N, and the Committee on Child Abuse and Neglect: Clinical report.

Guidance for the clinician rendering pediatric care. The evaluation of sexual abuse in children. Pediatrics 2005;116:506-512.

Klopfer U, Berger C, Lennertz I, Breuer B, Deget F, Wolke A, et al: Institutioneller $\checkmark$ Umgang mit sexuellem Missbrauch: Erfahrungen, Bewertungen und Wünsche nicht missbrauchender Eltern sexuell missbrauchter Kinder. Praxis Kinderpsychol Kinderpsychiatr 1999;48:647-663. 